\title{
Helios spacecraft data revisited: detection of cometary meteoroid trails by following in situ dust impacts
} \author{
Eberhard Grün $^{5,6}$, and Ralf Srama ${ }^{3,7}$ \\ ${ }^{1}$ Max-Planck-Institut für Sonnensystemforschung, Göttingen, Germany \\ e-mail: krueger@mps.mpg.de \\ 2 Planetary Exploration Research Center, Chiba Institute of Technology, Narashino, Japan \\ ${ }^{3}$ Institut für Raumfahrtsysteme, Universität Stuttgart, Germany \\ ${ }^{4}$ European Space Agency, European Space Astronomy Center, Madrid, Spain \\ ${ }^{5}$ Max-Planck-Institut für Kernphysik, Heidelberg, Germany \\ ${ }^{6}$ LASP, University of Colorado, Boulder, CO, USA \\ ${ }^{7}$ Baylor University, Waco, TX, USA
}

Harald Krüger ${ }^{1,2}$, Peter Strub ${ }^{1,3}$, Max Sommer ${ }^{3}$, Nicolas Altobelli ${ }^{4}$, Hiroshi Kimura ${ }^{2}$, Ann-Kathrin Lohse ${ }^{1}$,

Received 15 July 2020 / Accepted 21 September 2020

\begin{abstract}
Context. Cometary meteoroid trails exist in the vicinity of comets, forming a fine structure of the interplanetary dust cloud. The trails consist predominantly of the largest cometary particles (with sizes of approximately $0.1 \mathrm{~mm}-1 \mathrm{~cm}$ ), which are ejected at low speeds and remain very close to the comet orbit for several revolutions around the Sun. In the 1970s, two Helios spacecraft were launched towards the inner Solar System. The spacecraft were equipped with in situ dust sensors which measured the distribution of interplanetary dust in the inner Solar System for the first time. Recently, when re-analysing the Helios data, a clustering of seven impacts was found, detected by Helios in a very narrow region of space at a true anomaly angle of $135 \pm 1^{\circ}$, which the authors considered as potential cometary trail particles. However, at the time, this hypothesis could not be studied further.

Aims. We re-analyse these candidate cometary trail particles in the Helios dust data to investigate the possibility that some or all of them indeed originate from cometary trails and we constrain their source comets.

Methods. The Interplanetary Meteoroid Environment for eXploration (IMEX) dust streams in space model is a new and recently published universal model for cometary meteoroid streams in the inner Solar System. We use IMEX to study the traverses of cometary trails made by Helios.

Results. During ten revolutions around the Sun, the Helios spacecraft intersected 13 cometary trails. For the majority of these traverses the predicted dust fluxes are very low. In the narrow region of space where Helios detected the candidate dust particles, the spacecraft repeatedly traversed the trails of comets 45P/Honda-Mrkos-Pajdušáková and 72P/Denning-Fujikawa with relatively high predicted dust fluxes. The analysis of the detection times and particle impact directions shows that four detected particles are compatible with an origin from these two comets. By combining measurements and simulations we find a dust spatial density in these trails of approximately $10^{-8}-10^{-7} \mathrm{~m}^{-3}$.

Conclusions. The identification of potential cometary trail particles in the Helios data greatly benefited from the clustering of trail traverses in a rather narrow region of space. The in situ detection and analysis of meteoroid trail particles which can be traced back to their source bodies by spacecraft-based dust analysers provides a new opportunity for remote compositional analysis of comets and asteroids without the necessity to fly a spacecraft to or even land on those celestial bodies. This provides new science opportunities for future missions like DESTINY ${ }^{+}$(Demonstration and Experiment of Space Technology for INterplanetary voYage with Phaethon fLyby and dUst Science), Europa Clipper, and the Interstellar Mapping and Acceleration Probe.
\end{abstract}

Key words. comets: individual: 45P/Honda-Mrkos-Pajdusakova - comets: individual: 72P/Denning-Fujikawa - zodiacal dust meteorites, meteors, meteoroids - interplanetary medium - comets: general

\section{Introduction}

A cometary dust tail consists of small submicrometre-sized dust particles that are blown out by solar radiation pressure forces. Larger dust particles form the dust coma and later spread in the orbit of the comet as a result of small differences in orbital period. They form a tubular structure around the orbit of the parent comet called a dust trail. Dust trails in the vicinity of comets were first observed by the Infrared Astronomical Satellite (IRAS; Sykes et al. 1986), which identified a total of eight cometary meteoroid trails (Sykes \& Walker 1992). In subsequent infrared observations, at least $80 \%$ of the observed Jupiter-family comets were associated with dust trails which can thus be considered one of their generic features (Reach et al. 2007). More recently, detections of dust trails were also reported in the visible wavelength range (Ishiguro et al. 2007). A recent review of the present knowledge on cometary dust, including dust trails, was given by Levasseur-Regourd et al. (2018).

These trails form a fine structure superimposed on the interplanetary background dust cloud. They consist of the largest cometary particles (with sizes of approximately $0.1 \mathrm{~mm}-1 \mathrm{~cm}$; Agarwal et al. 2010), which are ejected at low speeds and remain very close to the comet orbit for several revolutions around the Sun. Trail particles are much bigger than the particles in the dust 
tail of the comet, and the latter disperse more rapidly as a result of higher ejection speeds and solar radiation pressure. When the Earth intercepts a cometary trail, the particles collide with the atmosphere and show up as meteors and fireballs (Koschny et al. 2019, and references therein). Effects of meteoroid impacts were also observed on the Earth Moon and on other planets (Christou et al. 2019). Until now there has been no known detection of a cometary trail with a spacecraft-based in situ dust detector.

In the 1970s, two Helios spacecraft were launched towards the inner Solar System. The goal of the missions was to reach an orbital perihelion at 0.3 AU from the Sun (Fig. 1), performing measurements of the interplanetary magnetic field, the solar wind, cosmic radiation, the zodiacal light, and the interplanetary dust distribution. The spacecraft were equipped with two in situ dust sensors each, which measured the distribution of interplanetary dust in the inner Solar System for the first time (Grün et al. 1980; Grün 1981).

Altobelli et al. (2006) re-analysed the Helios dust data searching for interstellar impactors (Grün et al. 1994; Krüger et al. 2019b). The authors recognised a cluster of seven impacts in a very narrow range of the spacecraft's true anomaly angle. Remarkably, these impacts were detected during a total of six Helios orbits at almost exactly the same spatial location. This coincidence led the authors to speculate that the impacts may have occurred during repeated spacecraft traverses of a cometary meteoroid trail. However, at the time, no detailed cometary trail model for the inner Solar System was available to further investigate this hypothesis.

Recently, the Interplanetary Meteoroid Environment for eXploration (IMEX) dust streams in space model was developed by Soja et al. (2015b,a, 2019) under contract by the European Space Agency. IMEX is a new universal and physical model for dust dynamics and orbital evolution that simulates recently created cometary dust trails in the inner Solar System. The IMEX model follows the trails of 420 comets and is ideal for studying meteor streams and cometary dust trails as measured by in situ detectors and observed in infrared images.

In this work, we use the IMEX model to investigate cometary trail traverses by the Helios spacecraft. We compare the measurements of seven candidate cometary trail particles identified by Altobelli et al. (2006) with trail traverses predicted by the model in order to investigate the hypothesis that a few or all of these particles originated from a cometary meteoroid trail. In Sect. 2, we briefly describe the Helios mission and the dust instruments on board, and in Sect. 3, we summarise the IMEX model. We present the results of our IMEX simulations and compare them with the Helios measurements in Sect. 4. In Sect. 5, we constrain the dust fluxes in the trails of two comets identified in our analysis. Section 6 presents a discussion and in Sect. 7 we present our outlook towards future perspectives. In Sect. 8, we summarise our conclusions.

\section{Helios dust measurements}

The Helios 1 spacecraft (we refer only to Helios 1 throughout this paper as the Helios 2 dust instruments did not provide useful dust measurements because of high noise rates on board the spacecraft) was launched into a heliocentric orbit on 10 December 1974. The Helios trajectory was in the ecliptic plane (inclination $i=0.02^{\circ}$ ). The eccentricity of the elliptical orbit was about $e=0.56$, the perihelion was located at $0.31 \mathrm{AU}$ from the Sun, the aphelion at $0.98 \mathrm{AU}$, and the argument of perihelion was $258.4^{\circ}$. The orbital period of Helios 1 was about 190 days, its orbit is shown in Fig. 1.

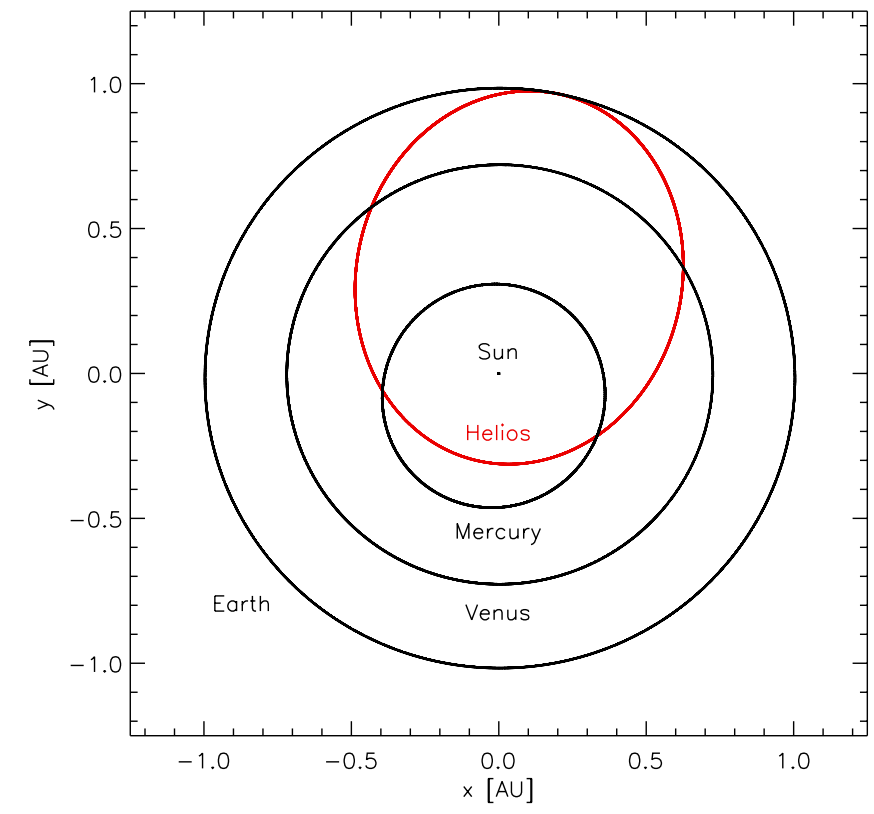

Fig. 1. Orbits of Helios (red), Mercury, Venus, and Earth. The $X-Y$ plane is the ecliptic plane with vernal equinox oriented towards the $+X$ direction.

The spacecraft was spin-stabilised with a spin axis pointing normal to the ecliptic plane and a spin period of one second. In Fig. 2, we show a schematic drawing of the spacecraft. It carried two dust instruments, the ecliptic sensor which was exposed to sunlight, and the south sensor which was shielded by the spacecraft from direct sunlight (Dietzel et al. 1973; Fechtig et al. 1978; Grün et al. 1980; Grün 1981). Between 19 December 1974 and 2 January 1980 the dust sensors transmitted the data of 235 dust impacts to Earth (Grün 1981). The true number of dust impacts onto the instruments was larger and missing data for these impacts is due to incomplete data transmission and instrumental dead-time (Grün et al. 1980).

The measurement principle of the Helios dust instruments was based on the impact ionisation generated upon impact of a high-velocity projectile onto a solid target (Dietzel et al. 1973; Auer 2001). From the measured signals, both the impact velocity and the mass of the impacting dust particle could be derived. In addition, the Helios instruments had time-of-flight mass spectrometer subsystems, providing information about the chemical elemental composition of the impactor. The target was a venetian blind consisting of gold strips held at ground potential. The constituents of the impact plasma were electrons, positive and negative ions, neutral atoms, or molecules, and residual fragments of the impactor and target. Electrostatic fields separated the positive and negative charges generated during the impact. The particle impact speed and mass were derived from both the rise time and the amplitude of the charge signals (Eichhorn 1978a,b; Grün et al. 1995). The Helios instruments had a detection threshold for dust particles with masses of approximately $3 \times 10^{-16} \mathrm{~kg}$ at an impact speed of $10 \mathrm{~km} \mathrm{~s}^{-1}$ (Grün et al. 1980).

The two Helios dust sensors were twin instruments. The socalled south sensor was sensitive to dust particles on inclined prograde heliocentric orbits. For an observer on board the spacecraft, those particles came from the ecliptic south direction. The second sensor was called ecliptic sensor since its field of view pointed towards the ecliptic plane. The field of view of each sensor was a cone with a half angle of $65^{\circ}$ (ecliptic sensor) 


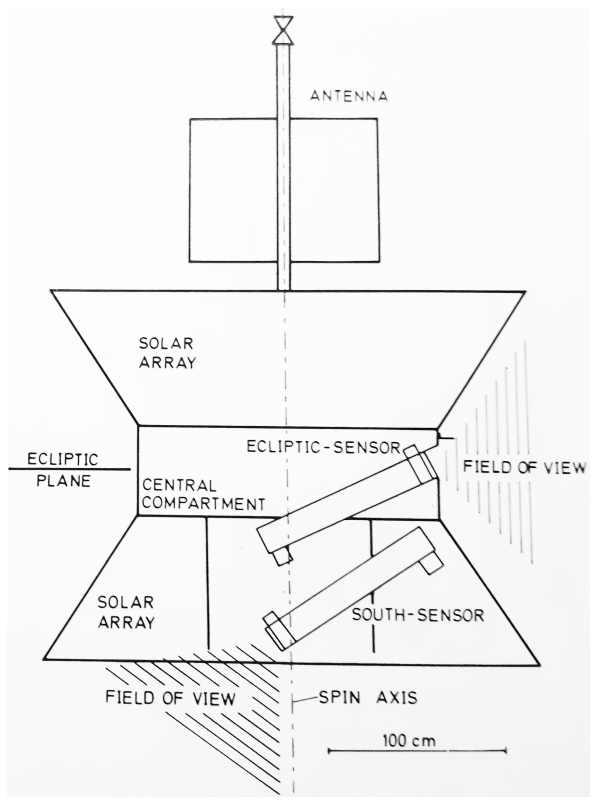

Fig. 2. Schematic drawing of the Helios spacecraft carrying the twin dust sensors. The ecliptic sensor was sensitive to dust particles on low inclined orbits, while the south sensor measured particles coming from the ecliptic south direction. Diagram adapted from Altobelli et al. (2006).

and $73^{\circ}$ (south sensor), respectively, centred on the sensor axis (Grün et al. 1980). Both instruments were partially shielded by the spacecraft structure, resulting in slightly different target areas: $54.5 \mathrm{~cm}^{2}$ for the ecliptic sensor, and $66.5 \mathrm{~cm}^{2}$ for the south sensor.

As the ecliptic sensor pointed into the Sun once per spacecraft rotation, an additional aluminium-coated parylene foil of $0.3 \mu \mathrm{m}$ thickness covered the instrument aperture. This foil prevented solar radiation from entering the sensor and heating it up beyond safe operations, but dust impactors could penetrate it. However, the sensitivity of the sensor was decreased. In contrast, the south sensor had only a protection against the solar wind plasma, which did not decrease its sensitivity.

The ecliptic sensor was sensitive to dust particles approaching with elevations from $-45^{\circ}$ to $+55^{\circ}$ with respect to the ecliptic plane. The south sensor could detect particles with trajectory elevations from $-90^{\circ}$ (ecliptic south-pole) to $-4^{\circ}$. During one spin revolution of the spacecraft, both instruments scanned an entire circle along the ecliptic plane. More details about the instruments and their calibration can be found in Grün et al. (1980); Grün (1981) and Altobelli et al. (2006).

Altobelli et al. (2006) re-analysed the Helios dust data, searching for interstellar particle impacts in the inner Solar System. When analysing the data as a function of Helios' true anomaly angle $\eta$, the authors recognised a cluster of seven impacts in a very narrow range $\eta=135 \pm 1^{\circ}$. Figure 3 shows a subset of the Helios dust data together with these cometary trail particle candidates. These data were obtained during a total of ten Helios orbits around the Sun.

The particle concentration at $\eta=135^{\circ}$ is indicated by a vertical solid line. These seven impacts were detected during six Helios orbits in a very narrow spatial range between 0.72 and $0.75 \mathrm{AU}$ distance from the Sun (two impacts occurred on the same day). The derived particle masses were in the range $10^{-16} \mathrm{~kg} \lesssim m \lesssim 10^{-12} \mathrm{~kg}$, with an uncertainty of a factor of 10 in the mass calibration of a single particle. This remarkable

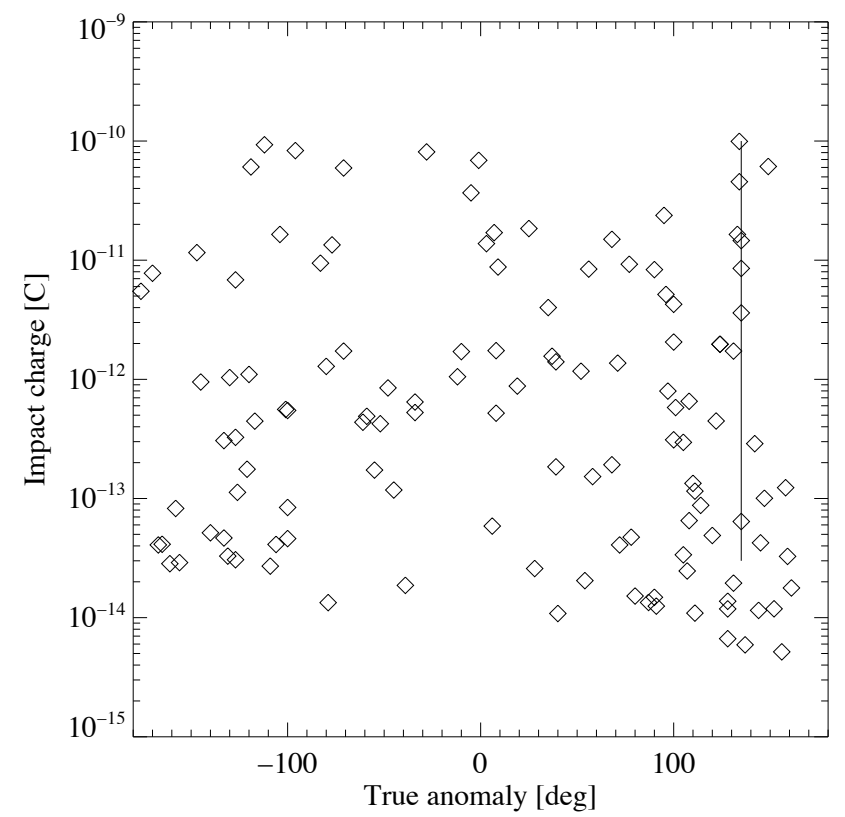

Fig. 3. Impact charges detected by the ecliptic and the south sensor as a function of Helios' true anomaly angle $\eta$ for a subset of the Helios dust data, from Altobelli et al. (2006). The vertical line shows a cluster of seven impacts which are candidates for cometary trail particles detected when the spacecraft intercepted one or more cometary trails.

coincidence of repetitive detections at approximately the same location led the authors to speculate that the impacts may have occurred when the Helios spacecraft repeatedly traversed the meteoroid trail of a comet. The authors argued that owing to their size, such grains would be little sensitive to radiation pressure, and they would keep the orbital elements of their parent body for some time. However, the hypothesis could not be investigated further because no comprehensive dust trail model was available at the time. Here, we study this hypothesis further.

\section{IMEX cometary trails model}

In order to identify time intervals when the Helios spacecraft traversed cometary meteoroid trails, we use the IMEX dust streams in space model developed by Soja et al. (2015b,a, 2019). The model generates trails for 362 Jupiter-family, 40 Halley-type, and 18 Encke-type comets available in the JPL Small Body Database (SBDB) as of 1 August 2013, which have perihelion distances $q<3 \mathrm{AU}$, semimajor axes $a<30 \mathrm{AU}$, and defined total visual magnitudes.

Particles are emitted when the comet is in the inner Solar System, taking into account comet perihelion passages between the years 1700 and 2080 for Encke-type comets, and between 1850 and 2080 for Jupiter-family and Halley-type comets, respectively. This reflects the fact that the most recent dust is expected to be most important, and also the maximum size of the database that could be maintained at the time when the model was developed.

For each passage through the inner Solar System within $3 \mathrm{AU}$ of the Sun of each comet (which we refer to apparitions in the following), particles are emitted randomly from the sunlit hemisphere of the comet nucleus within the time ranges specified above. About 28000 particles are ejected per comet per apparition for Halley-type comets; and this number is about 14000 for other comets. 
The dust ejection is described by the velocity model from the hydrodynamic comet emission model of Crifo \& Rodionov (1997). The model assumes the dust emission to be driven by water gas production within $3 \mathrm{AU}$ of the Sun.

The model estimates the water production rate using the visual magnitude, and a gas-to-dust ratio based on an empirical formula given by Jorda et al. (2008). The JPL Small Body Database provides total and nuclear magnitudes.

Dust-to-gas mass ratios can be estimated for individual comets, and they mostly range from 0.1 to 3 , though higher values are possible. Furthermore, they appear to be dependent on heliocentric distance (A'Hearn et al. 1995). Given the large uncertainties in dust-to-gas ratios, the model uses a value of 1. Deviations from this can be considered in the analysis of individual comets.

The IMEX model uses the mass distribution model of Divine \& Newburn (1987) and Agarwal et al. (2007, 2010), with model parameters given by Soja et al. (2015b). The mass distribution covers the range from $10^{-8} \mathrm{~kg}$ to $10^{-2} \mathrm{~kg}$, separated into eight mass bins (approximately corresponding to $100 \mu \mathrm{m}$ to $1 \mathrm{~cm}$ particle radius; Soja et al. 2015b). The particle density is assumed to be $\rho=1000 \mathrm{~kg} \mathrm{~m}^{-3}$. For comets with unknown radius a value of $1 \mathrm{~km}$ is assumed (Soja et al. 2015b).

The trajectory of each emitted particle is integrated individually including solar gravity, planetary perturbations, solar radiation pressure and Poynting-Robertson drag. Non-gravitational cometary forces are neglected because they are not well known for most comets, and their effect is largely to alter the location of the comet in its orbit rather than the orbit itself.

Due to storage space considerations, the particle state vectors are saved only during a limited time interval from 1980 to 2100 . In order to compare the IMEX model to the Helios data from 1975 to 1980 , we identified candidate comets by extrapolating the simulated particle state vectors backwards from 1980 using only solar gravity and radiation pressure, subsequently re-doing the full integration for the two comets with the highest flux, that is, 45P/Honda-Mrkos-Pajdušáková (hereafter 45P/H-M-P) and 72P/Denning-Fujikawa (hereafter 72P/D-F), and storing their particle state vectors starting from 1960.

The model calculates the impact velocity for each individual particle onto the spacecraft as well as dust number density and flux. We use the IMEX model to identify time intervals when Helios traversed the meteoroid trails of comets between December 1974 and January 1980 when dust measurements are available. A detailed model description including an application to the trail of comet 67P/Churyumov-Gerasimenko was given by Soja et al. (2015b).

\section{Results}

In this section, we present the results of our dust trail simulations for the time period between 19 December 1974 and 2 January 1980 when Helios collected dust measurements. In this time interval, the spacecraft completed ten revolutions around the Sun and repeatedly traversed the meteoroid trails of several comets. We compare the times when Helios detected the particles and the measured impact directions with the model predictions in order to constrain the particle sources.

\subsection{Simulated dust fluxes}

In Fig. 4, we show the simulated fluxes for Helios' cometary trail traverses. The simulations identified the trails of 13 comets that were traversed by Helios. The predicted fluxes for most of these crossings are below approximately $10^{-4} \mathrm{~m}^{-2} \mathrm{day}^{-1}$, which is insignificant for our analysis (for some comets the predicted flux is even below $10^{-7} \mathrm{~m}^{-2} \mathrm{day}^{-1}$ and therefore not shown in the diagram). The maximum dust fluxes predicted for trail traverses of individual comets vary by up to four orders of magnitude.

A repetitive pattern is obvious for comets $72 \mathrm{P} / \mathrm{D}-\mathrm{F}$ (red squares) and 45P/H-M-P (blue triangles): strong peaks occur during consecutive revolutions of Helios around the Sun. The model predicts maximum fluxes of approximately $3 \times$ $10^{-2} \mathrm{~m}^{-2}$ day $^{-1}$ for these two comets. The flux peaks are rather narrow with a typical peak width of approximately 5-20 days. Both are Jupiter family comets with orbital periods of 5 and 9 years, respectively (Table 1). Interestingly, for both comets the flux predicted for each trail traverse decreases with time for consecutive traverses (Fig. 4). Helios' trail traverses occurred soon after these two comets passed through their perihelia (Table 1), and this decreasing flux is in agreement with a drop in the dust density along the trail with increasing distance from the comet nucleus.

At the top of Fig. 4, we indicate the detection times of the seven dust particles at a true anomaly angle of $\eta=135 \pm 1^{\circ}$, which Altobelli et al. (2006) recognised as candidate trail particles. The simulations show that close to all these detections Helios traversed at least one meteoroid trail. This is particularly evident for comet 72P/D-F: a flux exceeding $6 \times 10^{-3} \mathrm{~m}^{-2} \mathrm{day}^{-1}$ is predicted for three trail traverses in December 1978, June 1979, and January 1980. Helios detected one particle impact during or close to each of these traverses.

There are also particle detections at the trail traverses of comet 45P/H-M-P in the interval 1975 to 1977 . Two particles were detected during the traverse of the trail of this comet in May 1976. On the other hand, there are traverses of the trail of this comet in 1976 and 1977 at a true anomaly angle $\eta \approx 225^{\circ}$ where there is no obvious particle concentration in the Helios data set (cf. Fig. 3). However, at this location the model predicts somewhat lower fluxes, making particle detections less likely, although the detection of single unidentified trail particles by Helios cannot be excluded. Figure 4 also shows that the seven candidate trail particles were particularly detected during trail traverses with the highest predicted dust fluxes.

In Fig. 5, we compare in more detail the detection times of the candidate trail particles with the time intervals when the model predicts the highest fluxes for comets 45P/H-M-P and 72P/D-F. Three detections in 1975 and 1976 nicely coincide with the time interval when the model predicts relatively high fluxes in the range of $10^{-2 \ldots-3} \mathrm{~m}^{-2} \mathrm{day}^{-1}$ (top panels). The two detections in 1979 and 1980 (bottom panels) are offset from the highest predicted trail fluxes by only one day. However, in two cases the offset is 4 days (1978) and 8 days (1977), respectively (middle panels). We analyse the particle detection times in more detail in Sect. 6.

\subsection{Detection geometry and impact speeds}

In addition to the detection time of the particles, the impact direction is another important parameter to constrain the origins of the particles. In Fig. 6, we show the Helios trajectory together with orbital sections for the comets that exhibit the highest meteoroid fluxes during trail traverses as shown in Fig. 4. The simulated impact directions of particles onto the spacecraft in the spacecraft-centric reference frame are indicated at a true 
H. Krüger et al.: Helios detections of cometary dust trails in the inner solar system

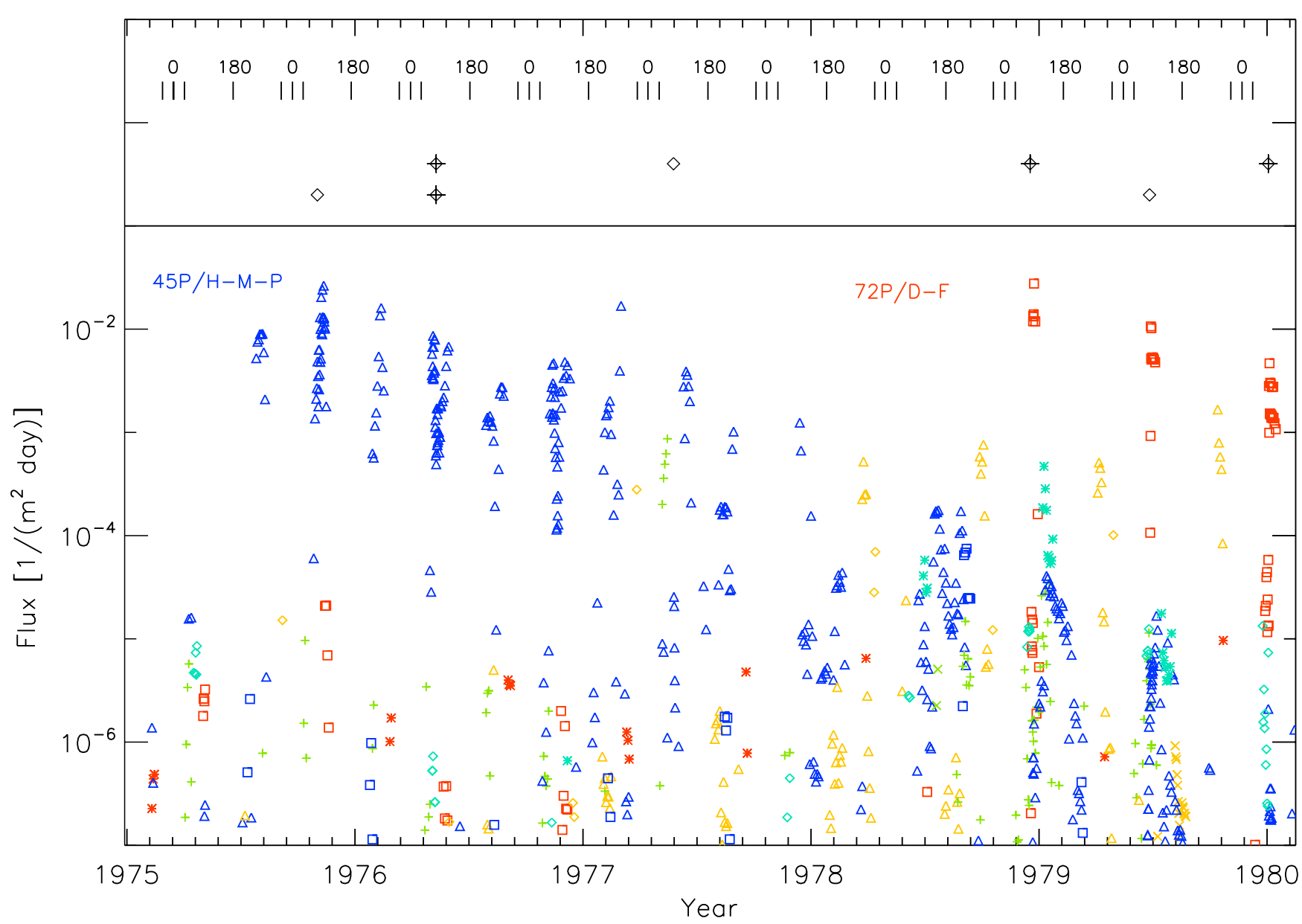

Fig. 4. Simulated dust fluxes for cometary meteoroid trails intercepted by the Helios spacecraft (cf. Fig. 6). Symbols and colours distinguish individual comets. Helios' true anomaly angle is indicated at the top. Black diamonds show the detection times of seven particles at a true anomaly angle of $\eta=135 \pm 1^{\circ}$. Four particles identified in this work as potential cometary trail particles are additionally marked with crosses (top row: detections with the ecliptic sensor. Bottom row: detections with the south sensor). The coloured symbols refer to the following comets: red squares: 72P/D-F; blue triangles: 45P/H-M-P; green crosses: 15P/Finlay; light blue asterisks: 141P/Machholz 2-A; yellow triangles: 210P/Christensen. The remaining symbols refer to other comets forming a very low background flux. The simulations were performed with a two-day time-step, and the simulated particles are in the mass range $10^{-8} \mathrm{~kg} \leq m \leq 10^{-2} \mathrm{~kg}$.

Table 1. Orbital data of comets discussed in this paper from the JPL Small Body Database (ssd. jpl . nasa.gov) unless stated otherwise, as well as the simulated approximate particle impact speed $v_{\text {imp }}$ at $\eta=135^{\circ}$ (Col. 9).

\begin{tabular}{|c|c|c|c|c|c|c|c|c|}
\hline $\begin{array}{c}\text { Comet } \\
\text { (1) }\end{array}$ & $\begin{array}{l}e \\
(2)\end{array}$ & $\begin{array}{c}q \\
\text { (AU) } \\
(3)\end{array}$ & $\begin{array}{c}i \\
\left({ }^{\circ}\right) \\
(4)\end{array}$ & $\begin{array}{l}\Omega \\
\left({ }^{\circ}\right) \\
(5)\end{array}$ & $\begin{array}{l}\omega \\
\left({ }^{\circ}\right) \\
(6)\end{array}$ & $\begin{array}{c}t_{\text {Perihelion }} \\
\text { (7) }\end{array}$ & $\begin{array}{c}\text { Epoch } \\
\text { (8) }\end{array}$ & $\begin{array}{c}v_{\mathrm{imp}} \\
\left(\mathrm{km} \mathrm{s}^{-1}\right) \\
(9)\end{array}$ \\
\hline 45P/Honda-Mrkos-Pajdušáková & 0.81 & 0.58 & 13.1 & 233.7 & 184.5 & 28-Dec.-1974 & 19-Dec.-1974 & 32 \\
\hline 72P/Denning-Fujikawa & 0.82 & 0.78 & 9.2 & 36.1 & 337.9 & 02-Oct.-1978 ${ }^{(a)}$ & 20-Nov.-2014 & 21 \\
\hline 15P/Finlay & 0.70 & 1.10 & 3.65 & 42.4 & 322.2 & 03-Jul.-1974 & 12-Jul.-1974 & 20 \\
\hline 141P/Machholz 2-A & 0.75 & 0.75 & 12.8 & 246.2 & 149.3 & 18-Sep.-1994 & 05-Sep.-1994 & 24 \\
\hline
\end{tabular}

Reference. ${ }^{(a)}$ Sato \& Williams (2014)

anomaly angle $\eta=135^{\circ}$, corresponding to the trail traverses for which the model predicts the highest dust fluxes (cf. Fig. 4). Orbital elements for these comets are listed in Table 1.

For the relevant comets, the simulated particle impact speeds are between $20 \mathrm{~km} \mathrm{~s}^{-1}$ and $32 \mathrm{~km} \mathrm{~s}^{-1}$ (Table 1). The measured particle impact speeds are in the range of approximately $10-40 \mathrm{~km} \mathrm{~s}^{-1}$ (Table 2), which is in rather good agreement with these values, given that the single speed measurement has an uncertainty of at least a factor of two.

Figure 6 shows that by remarkable coincidence, at a true anomaly angle of approximately $\eta=135^{\circ}$, Helios traversed the trails of three comets: 45P/H-M-P, 72P/D-F and 141P/Machholz 2-A, with the orbit of a fourth one, 15P/Finlay, also being close. Furthermore, the traverse of Venus' orbit occurred in the same 

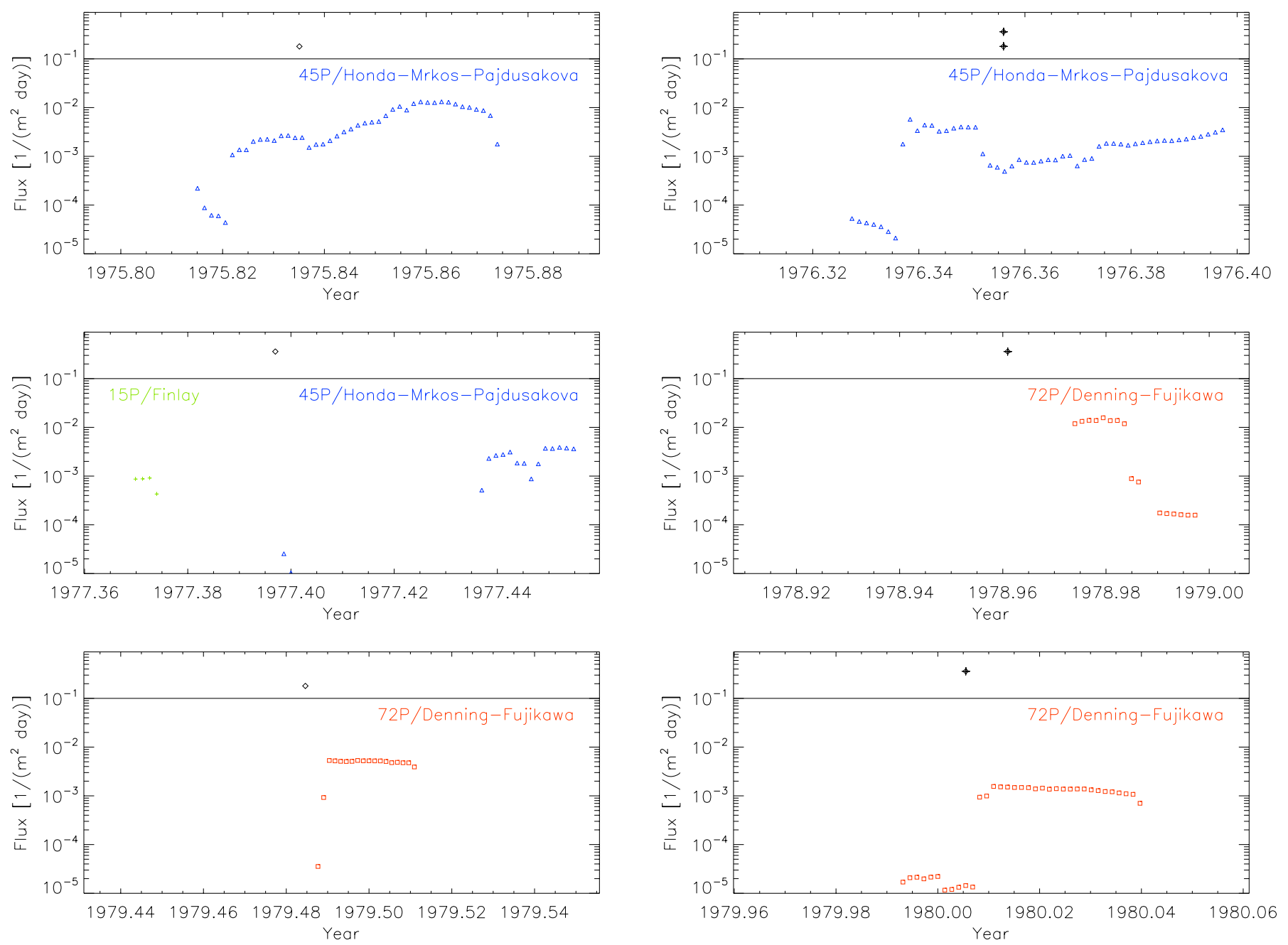

Fig. 5. Same as Fig. 4 but for the time periods when Helios detected the candidate cometary trail particles. For each panel, the time interval shown is one month and the separation of each simulated data point is $12 \mathrm{~h}$. The times of the Helios detections are indicated at the top.

region. This coincidence suggests that the candidate trail particles could be particularly easily recognised as such because of this concentration of trail traverses within a rather small region of space.

From the spacecraft spin orientation at the time of dust detection we can constrain the impact direction of each detected particle. In Fig. 7, we compare the Helios detections with the simulated impact directions for trail particles released from comets 72P/D-F and 45P/H-M-P. Here the largest uncertainties arise from the rather large sensor field of view (indicated by red crosses) while the spacecraft orientation is known with a high accuracy of better than $1.4^{\circ}$. Some fine structure is evident in the simulated trails. The geometries for 141P/Machholz 2-A and 15P/Finlay are very similar, however they are not considered further because for them the model predicts significantly lower fluxes than for the other two comets (cf. Fig. 4).

In Table 2 we summarise our results for the particle detections. The strongest criterion is the impact direction. If this is not compatible with a trail origin we discard the particle from the list of potential trail particles. Figure 7 shows that this is the case for the three detections in 1975, 1977, and 1979. From the remaining four particles, the particle measured in 1978 is compatible with an origin from comet $72 \mathrm{P} / \mathrm{D}-\mathrm{F}$, while the three detections in 1976 and 1980 are marginally compatible with an origin from comets $72 \mathrm{P} / \mathrm{D}-\mathrm{F}$ and $45 \mathrm{P} / \mathrm{H}-\mathrm{M}-\mathrm{P}$, respectively. The impact times agree with this interpretation for the two detections in 1976, while there is an offset for the detections in 1978 and 1980. The impact speed is only listed in Table 2 for comparison, it is not used as a criterion for trail identification.

The fields of view of the Helios dust sensors are shown in Fig. 8. For the dust impacts measured in 1976, 1978, and 1980, which are in best agreement with a cometary trail origin, we also show the impact directions of the simulated trails of comets 45P/H-M-P and 72P/D-F, as well as the approximate directions of Venus dust ring particles orbiting the Sun on circular heliocentric orbits. As was already concluded from Fig. 7, the particle detected in 1978 was well within the dust sensor field of view while the three other detections were likely close to the edge of the field of view. The sensor side wall is not taken into account in Fig. 8 (cf. Sect. 6).

It should be emphasised that for our analysis we only used directly measured parameters, such as for example the sensor azimuth and the spacecraft true anomaly angle at the time of particle impact. We only refer to derived physical parameters such as impact speed and mass to check for consistency with our simulation results. Therefore, our analysis is free of any uncertainties of the type introduced by empirical calibrations applied to derive these physical parameters from the measured quantities. 
Table 2. Data for the candidate cometary trail particles considered in this work.

\begin{tabular}{|c|c|c|c|c|c|c|c|c|c|c|}
\hline \multirow{2}{*}{$\begin{array}{l}\text { Day } \\
\text { (1) }\end{array}$} & \multirow{2}{*}{$\begin{array}{l}\text { Sensor } \\
\text { (2) }\end{array}$} & \multirow{2}{*}{$\begin{array}{c}\text { Measured } \\
\text { impact speed } \\
\left(\mathrm{km} \mathrm{s}^{-1}\right) \\
\text { (3) }\end{array}$} & \multirow{2}{*}{$\begin{array}{c}\text { Measured } \\
\text { particle mass } \\
\text { (kg) } \\
\text { (4) }\end{array}$} & \multicolumn{3}{|c|}{$\begin{array}{l}\text { Compatibility with trail origin } \\
\text { based on }\end{array}$} & \multirow{2}{*}{ 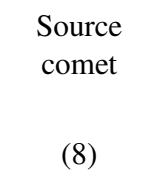 } & \multirow{2}{*}{$\begin{array}{l}\text { Simulated } \\
\text { impact speed } \\
\left(\mathrm{km} \mathrm{s}^{-1}\right) \\
\text { (9) }\end{array}$} & \multirow{2}{*}{$\begin{array}{c}\text { Trail flux } \\
\Phi \\
\left(\mathrm{m}^{-2} \mathrm{day}^{-1}\right) \\
(10)\end{array}$} & \multirow{2}{*}{$\begin{array}{c}\text { Dust density } \\
D \\
\left(\mathrm{~m}^{-3}\right) \\
(11)\end{array}$} \\
\hline & & & & $\begin{array}{c}\text { Time } \\
(5)\end{array}$ & $\begin{array}{l}\text { Direction } \\
(6)\end{array}$ & $\begin{array}{l}\text { Speed } \\
(7)\end{array}$ & & & & \\
\hline 75-11-01 & S & $100_{-50}^{+100}$ & $5.1_{-4.6}^{+46} \times 10^{-20}$ & Yes & No & - & - & - & - & - \\
\hline 1976-05-09 & S & $11_{-5}^{+8}$ & $5.4_{-4.6}^{+375} \times 10^{-13}$ & Yes & Possibly & (No) & \multirow{2}{*}{ 45P/H-M-P } & \multirow{2}{*}{$33 \pm 1.8$} & \multirow{2}{*}{$\approx 67$} & \multirow{2}{*}{$\approx 2 \times 10^{-8}$} \\
\hline 1976-05-09 & E & $34_{-15}^{+26}$ & $7.1_{-6.1}^{+493} \times 10^{-15}$ & Yes & Possibly & (Yes) & & & & \\
\hline $1977-05-25$ & $\mathrm{E}$ & $18_{-8}^{+14}$ & $1.5_{-1.3}^{+8.5} \times 10^{-15}$ & Possibly & No & - & - & - & - & - \\
\hline $1978-12-17$ & $\mathrm{E}$ & $9_{-4}^{+6}$ & $2.2_{-1.9}^{+13} \times 10^{-16}$ & Possibly & Yes & (No) & 72P/D-F & $21 \pm 1.9$ & $\lesssim 158$ & $\lesssim 9 \times 10^{-8}$ \\
\hline 1979-06-26 & S & $39_{-15}^{+26}$ & $6.9_{-5.9}^{+41} \times 10^{-17}$ & Possibly & No & - & - & - & - & \\
\hline 1980-01-02 & $\mathrm{E}$ & $3_{-2}^{+1}$ & $1.6_{-1.4}^{+9.5} \times 10^{-12}$ & Possibly & Possibly & (No) & 72P/D-F & $21 \pm 2.5$ & $\lesssim 158$ & $\lesssim 9 \times 10^{-8}$ \\
\hline
\end{tabular}

Notes. Columns: are detection day, sensor that detected the particle, measured impact speed, measured particle mass, impact speed derived from IMEX simulation, compatibility with trail origin based on given parameters, source comet, flux in trail from source comet. Measured data in Cols. 1-4 are from Grün (1981).

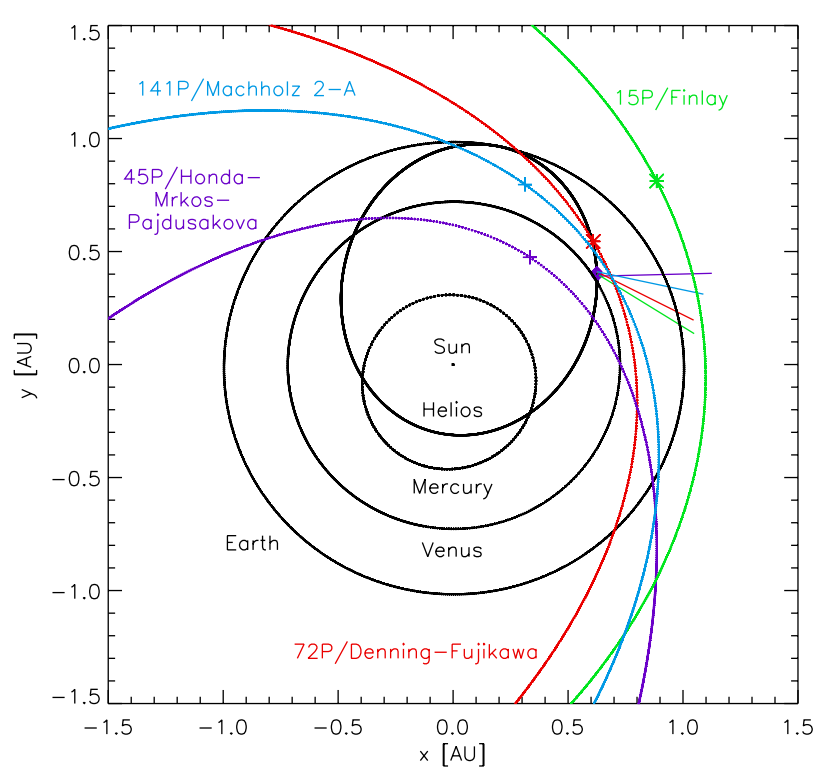

Fig. 6. Orbits of Helios and the candidate comets. The purple diamond marks the dust detections at $\eta \approx 135^{\circ}$. The lines attached to the diamond indicate the approximate impact directions (speed vector) of trail particles from these comets in the spacecraft-centric reference frame as derived from the IMEX model. The $X-Y$ plane is the ecliptic plane with vernal equinox oriented towards the $+X$ direction. Comet orbits are shown for the period 1975-1980, with locations of ascending nodes (asterisks) and descending nodes (plus signs) superimposed.

\subsection{Mass spectra}

In addition to impact speed and particle mass, the Helios dust instruments measured the particle composition with low mass resolution. The mass spectra of the seven candidate cometary trail particles are shown in Fig. 9. We discuss them in more detail in Sect. 6.

\section{Estimation of dust fluxes from the measurements}

Our IMEX trail simulations show that up to four of the seven candidate cometary trail particles detected by Helios are compatible with an origin from comet $45 \mathrm{P} / \mathrm{H}-\mathrm{M}-\mathrm{P}$ or $72 \mathrm{P} / \mathrm{D}-\mathrm{F}$. Based on these trail identifications we attempt to constrain the dust fluxes in these trails by combining measurements and model results.

The case of a single particle detection with an in situ dust detector was considered by Hirn et al. (2016). The authors applied Poisson statistics to the measurements performed by the Dust Impact Monitor (DIM) on board the Rosetta lander Philae at comet 67P/Churyumov-Gerasimenko. The DIM detected a single particle impact during the descent of Philae to the comet surface (Krüger et al. 2015). Here we apply a similar approach to our Helios detections.

We assume that the cometary trail is a closely collimated stream of particles and that the impacts onto the Helios sensors are independent events, and therefore that they follow a Poisson distribution. For the periods when exactly one impact was detected during a trail traverse, only an upper limit for the ambient trail flux can be estimated. We define the upper limit of the expected number of impacts as the highest value of $\lambda$ for which there is an arbitrarily chosen $5 \%$ probability that the number of detected events $N$ is less than two in a single measurement:

$$
\begin{aligned}
P(N<2) & =P(N=0)+P(N=1) \\
& =\frac{\lambda^{0} \exp (-\lambda)}{0 !}+\frac{\lambda^{1} \exp (-\lambda)}{1 !} \\
& =(1+\lambda) \exp (-\lambda)=0.05,
\end{aligned}
$$

resulting in

$\lambda \approx 4.74$.

The maximum impact rate is

$N_{\text {max }}=\lambda_{\text {max }} / T_{\text {meas }}$,

where $\lambda_{\max }$ is given by Eq. (2) and $T_{\text {meas }}$ is the measurement time. For $T_{\text {meas }}$ we assume the duration of a trail traverse predicted by the model which is typically 10 days (cf. Sect. 4.1). The maximum flux onto the sensors is given by

$\Phi_{\text {max }}=\frac{N_{\text {max }}}{A}=\frac{\lambda_{\text {max }}}{T_{\text {meas }} A}$,

where $A$ is the spin-averaged effective sensor area. 

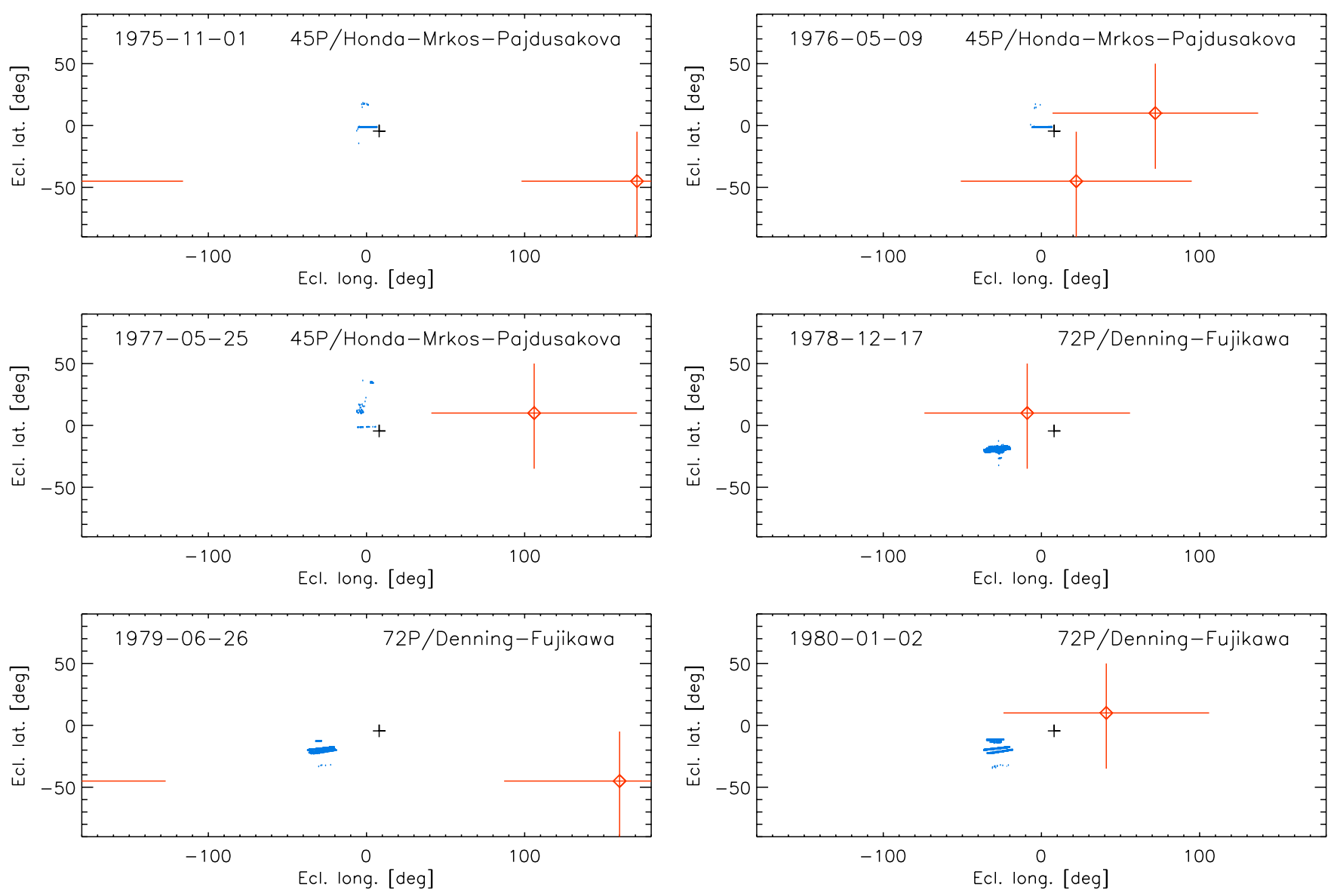

Fig. 7. Impact directions of detected particles compared with the directions of simulated trails for comets 72P/D-F and 45P/H-M-P in the spacecraftcentric reference frame in ecliptic coordinates. Blue dots show the approach directions of simulated trail particles. Red diamonds indicate the sensor orientation during the time of particle impact; the ecliptic longitude of the data point is the spacecraft spin orientation at the time of particle impact while the ecliptic latitude corresponds to the maximum of the sensor sensitivity profile in latitudinal direction (Grün et al. 1980). Red crosses indicate the approximate sensor field of view (cf. Fig. 8), and small black crosses show the impact direction of particles orbiting the Sun on circular orbits at the heliocentric distance of Venus. The detection time of the impact is given in the top left corner of each panel.

Finally, the dust spatial density $D$ is given by

$D_{\max }=\frac{\Phi_{\max }}{v_{\text {imp }}}$

where $v_{\text {imp }}$ is the impact speed of the particles.

Grün et al. (1980) give sensor areas of $54.5 \mathrm{~cm}^{2}$ for the ecliptic sensor, and $66.5 \mathrm{~cm}^{2}$ for the south sensor. Given that both sensors were always operated simultaneously, we simply add the two areas to obtain a total area of $121 \mathrm{~cm}^{2}$ for both sensors together. Due to the spacecraft spin, the spin-averaged effective sensor area was about a factor of four smaller, that is, $A \approx 30 \mathrm{~cm}^{2}$. With these numbers Eq. (4) gives an upper limit for the dust flux in the trail of comet $72 \mathrm{P} / \mathrm{D}-\mathrm{F}$ :

$\Phi_{\max , 72 \mathrm{P}}=158 \mathrm{~m}^{-2} \mathrm{day}^{-1}$,

and with the impact speed derived from the model given in Table 2, the upper limit for the dust spatial density becomes

$D_{\max , 72 \mathrm{P}}=9 \times 10^{-8} \mathrm{~m}^{-3}$.

These upper limits apply to the cases when Helios detected one single particle per trail traverse.
Similarly, for the case when two potential trail particles were detected during the traverse of the trail of comet 45P/H-M-P in 1976, we get a flux of

$\Phi_{45 \mathrm{P}}=\frac{N}{A}=\frac{2}{10 \text { days } \times 0.003 \mathrm{~m}^{2}}=67 \mathrm{~m}^{-2} \mathrm{day}^{-1}$,

and a dust density of

$D_{45 \mathrm{P}}=2 \times 10^{-8} \mathrm{~m}^{-3}$.

At first glance, these fluxes seem to be very high, and they are indeed three to four orders of magnitude larger than the values predicted by the model. However, one has to take into account the fact that the model predicts the fluxes of particles of about $100 \mu \mathrm{m}$ in size and bigger while Helios detected particles at least a factor of ten smaller, and our flux estimates refer to these smaller particles. If we assume a dust size distribution following a power law with a differential exponent of approximately -4 (Agarwal et al. 2010, references therein) and extend this to the approximately $10 \mu \mathrm{m}$ particles as implied by the Helios measurements, our derived flux values are in reasonable agreement with the simulated fluxes.

We do not consider any statistical uncertainty here because systematic effects most likely lead to much larger uncertainties. For example, our calculation assumes that the particles were 
H. Krüger et al.: Helios detections of cometary dust trails in the inner solar system
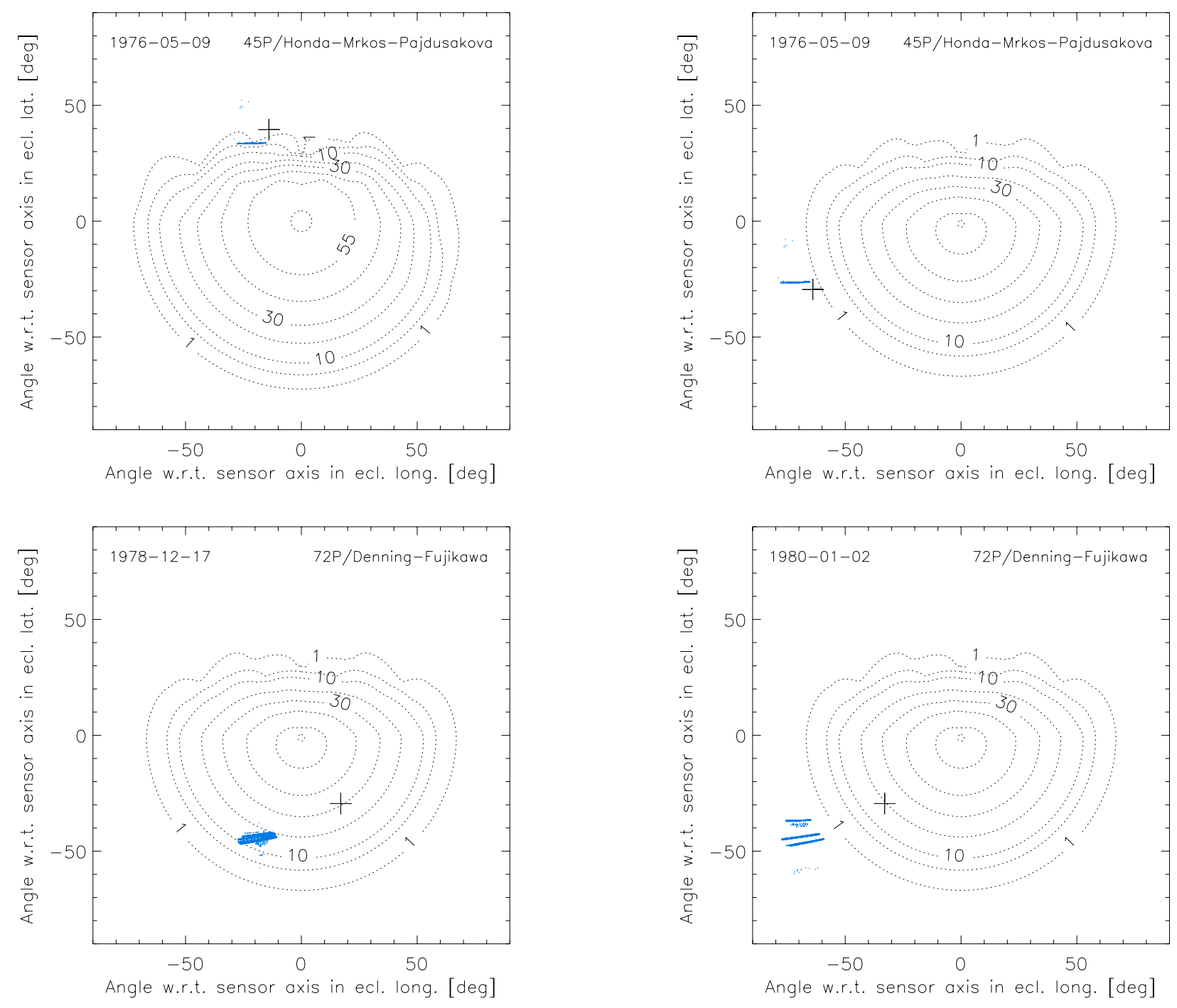

Fig. 8. Sensor field of view for the south sensor (top left panel) and the ecliptic sensor (all other panels) from Grün et al. (1980). The centre (coordinates $[0,0]$ ) is the direction of the sensor boresight. The numbers at the contour lines give the sensitive area of the sensor target in square centimetres, which depends on the particle impact direction. The simulated impact directions of the cometary trail particles (blue dots), and particles orbiting the Sun on circular orbits at Venus' orbit (black cross) are superimposed for the sensor pointing at the impact time of the dust particle; see also Fig. 7.

detected with a sensor orientation represented by the maximum of the sensor area $A$. However, Fig. 7 shows that this is most likely not the case for most of the detections. Instead, detections close to the edge of the field of view are more likely, which would imply significantly higher fluxes. On the other hand, dustsensitive sensor side walls would increase the sensitive area and reduce the derived fluxes (Sect. 6). Other factors are the uncertainty in the spatial extent of the trail and the identification of trail particles in the Helios data. In conclusion, we expect that the dust flux estimates we performed here have an uncertainty of at least a factor of 10 .

Dust fluxes simulated by IMEX can generally be considered as lower limits for two reasons. First, the model simulates only particles larger than $10^{-8} \mathrm{~kg}$ (corresponding to approximately $100 \mu \mathrm{m}$ ), while the cometary trails most likely contain smaller particles as well (Agarwal et al. 2010). Given that such smaller particles are more susceptible to radiation pressure and Poynting-Robertson drag than the larger ones, they are more quickly dispersed from the orbit of the comet than the larger trail particles. Nevertheless, a fraction of the recently released small particles remain close to the trail for some time (cf. Sect. 6). Second, by comparing model results with cometary meteoroid stream observations, Soja et al. (2015b) concluded that the model likely underestimates the true fluxes of particles of $100 \mu \mathrm{m}$ and bigger by at least an order of magnitude.

\section{Discussion}

Our simulations give the best agreement with the particle detected by Helios in 1978; it may be a trail particle released from comet 72P/D-F. Three more particle detections show marginal agreement with a cometary trail origin from comets $72 \mathrm{P} / \mathrm{D}-\mathrm{F}$ (detection in 1980) and 45P/H-M-P (two detections in 1976). In Fig. 7, the big crosses indicate the fields of view of the Helios dust instruments, and these three impacts may have occurred close to the edge of the sensor field of view. The crosses represent the sensor targets including shielding by the spacecraft structure (Grün et al. 1980). The analysis of data obtained with the dust detectors on board the Galileo and Ulysses spacecraft, 

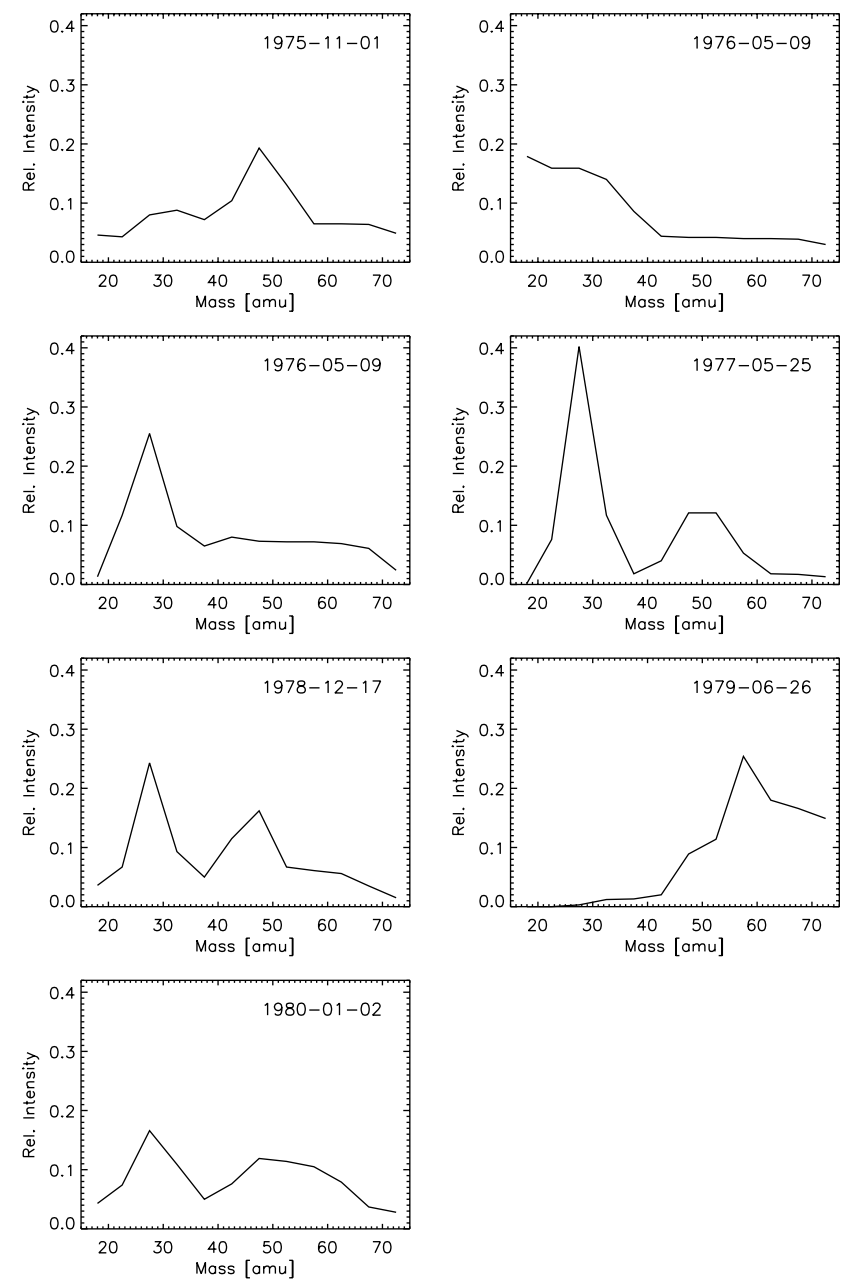

Fig. 9. Mass spectra of the seven dust particles detected at a true anomaly angle of $\eta=135 \pm 1^{\circ}$. The day when the impact occurred is given in the top right corner of each panel.

which were impact ionisation dust detectors of a similar design to the Helios instruments - except that they did not have the capability to measure time-of-flight spectra -, showed that their sensor side walls were sensitive to dust impacts as well (Altobelli et al. 2004). The sidewalls of the Helios sensors were made of metal, implying that they also had a high yield for impact ionisation, although this was never tested in the laboratory. Dustsensitive sidewalls would increase the sensor field of view and reduce the derived dust fluxes. The larger field of view would give much better agreement of the 1976 and 1980 dust impacts with a cometary trail origin.

Figure 5 shows an offset in the dust particle detections as compared to the times predicted by the model. This is particularly evident for the detection in 1978 which is offset from the time interval with the predicted highest dust fluxes by more than four days, corresponding to a spacecraft motion of approximately 0.04 AU. There are various possible reasons for such an offset:

First, the IMEX model simulates only particles with masses $m=10^{-8} \mathrm{~kg}$ and bigger, corresponding to particle radii above approximately $100 \mu \mathrm{m}$. The masses of the seven detected particles as derived from the calibration of the Helios dust instrument are at least four orders of magnitude smaller, corresponding to particle radii of a few micrometres to about $10 \mu \mathrm{m}$ (cf. Table 2). Such smaller particles are more susceptible to radiation pressure and Poynting-Robertson drag than the larger ones. For particles larger than approximately $10 \mu \mathrm{m}$ radius, the ratio of the force of solar radiation pressure $F_{\text {rad }}$ over that of gravity $F_{\text {grav }}$ is below $\beta \lesssim 0.05$ for most materials, while micrometre-sized and smaller particles may have values of $\beta>0.5$, and for some materials (e.g. metals) the value of $\beta$ can be even larger than one (Burns et al. 1979; Kimura \& Mann 1999). This indicates that the measured particles were more susceptible to radiation pressure and Poynting-Robertson drag than those simulated by the model. For example, the perihelion distance of a $10 \mu \mathrm{m}$ particle (with $\beta=$ 0.05 ) on an eccentric orbit with semi-major axis $a=3 \mathrm{AU}$ and eccentricity $e=0.7$ decreases by only approximately $0.0005 \mathrm{AU}$ within 100 years, while for a $100 \mu \mathrm{m}$ particle this drift is ten times smaller. This suggests that Poynting-Robertson drag alone cannot account for a significant particle drift on the timescales covered by the model. Second, the model uses a dust ejection model to simulate the dust emission from the comet nucleus due to water ice sublimation (Crifo \& Rodionov 1997; see also Soja et al. 2015b). Particles of $100 \mu \mathrm{m}$ (with density $1000 \mathrm{~kg} \mathrm{~m}^{-3}$ ) are ejected from the subsolar point on the surface of a nucleus with $1 \mathrm{~km}$ radius at $0.7 \mathrm{AU}$ heliocentric distance at about $80 \mathrm{~m} \mathrm{~s}^{-1}$. This is well above the escape speed from the nucleus. Smaller particles have higher ejection speeds. Although the detailed particle motion is also strongly affected by the ejection direction from the nucleus surface as well as solar radiation pressure and Poynting-Robertson drag, the particle ejection due to sublimation adds to the particle drift in the vicinity of the nucleus. Third, the model simulates the particle dynamics for only up to 300 years depending on the orbital period of the comet. This limitation was necessary based on the computing power available at the time in order to simulate the dynamics of a sufficiently large number of dust particles for all 420 comets covered by the model (Soja et al. 2015b). The previous considerations show that older particles are also likely to be detected further away from the nucleus. Finally, with increasing time, encounters with planets - Jupiter in particular - may significantly disturb the dust trails and move particles away from their source comet. One or more of these effects may explain the difference between the measured and the predicted particle detection time.

Particle masses given in Table 2 were derived from the calibration of the Helios dust instruments in the laboratory. Because of the venetian blind-type target there is a large spread in the recorded impact charge depending on where the impact occurred, that is, on the entrance side or on the multiplier side of the target strip. Furthermore, impacts on the sensor side wall may generate a very reduced impact charge. Therefore, the size (mass) of the impactors may be significantly underestimated, and even more so if cometary particles are fluffy aggregates, as indicated for example by the results from the Rosetta mission to comet 67P/Churymov-Gerasimenko (Güttler et al. 2019; Kimura et al. 2020b,a). This is also supported by the particle dynamics: particles released at perihelion from comets 72P/D-F and $45 \mathrm{P} / \mathrm{H}-\mathrm{M}-\mathrm{P}$, whose orbital eccentricity is $e=0.82$, cannot remain in a bound heliocentric orbit unless their $\beta$ ratios are smaller than 0.09 . This means that particles of approximately $5 \mu \mathrm{m}$ in radius and smaller are very quickly removed from these trails and escape from the Solar System on hyperbolic trajectories. For very porous particles with $85 \%$ porosity, consistent with Rosetta results, this limit increases to $3.0 \times 10^{-11} \mathrm{~kg}$, corresponding to a particle radius of about $14 \mu \mathrm{m}$. Therefore, if Helios really detected particles belonging to a cometary trail, they must have been significantly bigger than the sizes derived from the instrument calibration. Therefore, the chances are low that Helios rather observed micrometre-sized cometary trail particles. This is similar to the situation when Ulysses detected 
Jupiter stream particles (Grün et al. 1998): we only learned from modelling that the particles actually had nanometre sizes, and were much smaller than particle sizes derived from the instrument calibration.

Remarkably, between 1881 and 2014, comet 72P/D-F was only observed during its 1978 apparition. During all the other apparitions it was not re-discovered; although based on the observing conditions and predicted brightnesses, recoveries should have been possible a few times (Beech 2001; Sato \& Williams 2014). From these non-detections, the authors concluded that this comet may not have been active during all of its past apparitions. If $72 \mathrm{P} / \mathrm{D}-\mathrm{F}$ indeed had dormant periods in the past, the dust spatial density or the volume filled by the trail, or both, may be overestimated by the IMEX model. In a similar way, comet 15P/Finlay may also be evolving into a transitional asteroid-like object (Beech et al. 1999), although it possess the ability for repetitive energetic outbursts (Ishiguro et al. 2016).

The detections of our seven candidate cometary trail particles close to the orbit of Venus is intriguing (cf. Fig. 6). In addition to the in situ dust instruments, Helios also carried a zodiacal light photometer which discovered a heliocentric dust ring along Venus' orbit (Leinert \& Moster 2007). This ring was later confirmed by observations with the Heliospheric Imager instruments on board the two STEREO spacecraft (Solar Terrestrial Relations Observatory, Jones et al. 2013), and in situ measurements by the Arrayed Large-Area Dust Detectors in INterplanetary space (ALADDIN) on board the IKAROS spacecraft (Interplanetary Kite-craft Accelerated by Radiation Of the Sun) show a dust flux variation that may be connected with a Venusian dust ring (Hirai et al. 2014; Yano et al. 2014). From the STEREO observations, the enhancement in the dust spatial density in the Venus ring with respect to the interplanetary dust background was found to be only $8 \%$ at most (Jones et al. 2017). Interestingly, the STEREO observations showed a steplike increase in the dust density on the inner side of Venus' orbit while there was no drop in dust density detected on the outer side. Furthermore, dynamical modelling indicates that relatively small particles as measured by Helios $(\lesssim 10 \mu \mathrm{m})$ cannot be effectively trapped in resonances with Venus due to the stronger Poynting-Robertson drag and thus are unlikely to contribute to a dust enhancement in the Venus ring (Pokorný \& Kuchner 2019; Sommer et al. 2020). The relatively weak enhancement in dust density together with the required large particle sizes makes it unlikely that Helios detected impacts by Venus dust ring particles at $\eta=135^{\circ}$, although their impact speed and directions are in the same range as those of the cometary trail particles (Fig. 7; $\left.v_{\text {imp }}=17 \mathrm{~km} \mathrm{~s}^{-1} ; \lambda_{\text {ecl }}=8^{\circ}, \beta_{\text {ecl }}=-5^{\circ}\right)$.

Figure 6 reveals the remarkable coincidence that Helios traversed three known cometary trails at a true anomaly angle of $\eta \approx 135^{\circ}$. Because of this coincidence and high dust fluxes in the trails, Altobelli et al. (2006) were able to identify a concentration of seven dust impacts in the data of the relatively small Helios detectors. Figure 4 shows that if the spacecraft had traversed only one of the trails of 45P/H-M-P or 72P/D-F but not both at $\eta \approx 135^{\circ}$, Helios would only have reported three or four particle detections, respectively, at this true anomaly angle. It is unlikely that Altobelli et al. (2006) would have recognised such a small number of particles as potentially being of cometary trail origin.

By an interesting coincidence, from studying the orbital data of fireballs associated with the $\alpha$ Capricornids meteor stream in Earth's atmosphere, Hasegawa (2001) concluded that exactly our three candidate comets 45P/H-M-P, 72P/D-F , and 141P/Machholz 2-A could be associated with this meteor stream, out of six possible candidates including three additional comet and asteroid candidates. Comet $45 \mathrm{P} / \mathrm{H}-\mathrm{M}-\mathrm{P}$ was also predicted to be the source of a meteor shower in the Venus atmosphere (Vaubaillon \& Christou 2006).

The clustering of trail crossings at a true anomaly angle of $\eta \approx 135^{\circ}$ also explains why there was no particle concentration detected at approximately $\eta \approx 225^{\circ}$ even though the model predicts trail crossings here as well (cf. Fig. 4): first, the fluxes predicted by IMEX for single trails are somewhat lower than for the traverses at $\eta \approx 135^{\circ}$, and second, the cometary orbits are much more widely separated in space (Fig. 6).

Comet trails were first identified in the IRAS all sky survey (Sykes \& Walker 1992). Subsequently, in a survey of 34 Jupiterfamily comets with the Spitzer Space Telescope, at least $80 \%$ of the comets were associated with dust trails (Reach et al. 2007). Comet trails were also studied with the Diffuse Infrared Background Experiment (DIRBE) instrument on board the Cosmic Background Explorer (COBE; Arendt 2014) and with groundbased observations in the visible range (Ishiguro et al. 2007). Unfortunately, none of the comets identified in our analysis were contained in any of these surveys.

Radar observations of cometary comae can provide information about the particle sizes comprising the coma. Observations with the Arecibo Observatory planetary radar system showed that the coma of comet 45P/H-M-P contains particles larger than $2 \mathrm{~cm}$ (Springmann et al. 2017), while the existence of smaller particles could not be excluded.

Particle mass spectra can provide valuable information about the composition and evolution of the source bodies of the detected particles. Only two interplanetary dust particles were successfully analysed with the Cosmic Dust Analyzer (CDA; Srama et al. 2004) on board the Cassini spacecraft during its journey to Saturn (Hillier et al. 2007). Surprisingly, both particles had a very similar metallic (iron) composition with an absence of typical features expected for silicate minerals (e.g. silicon). The authors concluded that the particles were compatible with an asteroidal origin, although an origin from Jupiter family comets would also be possible. A few of the Helios spectra shown in Fig. 9 also show a broad feature covering iron (56 amu). In particular, the mass spectrum of the particle detected in 1979 is very similar to the CDA detections. Furthermore, at least five additional Helios particles (both in 1976, 1977, 1978 and 1980) show a broad peak covering silicon ( $28 \mathrm{amu}$ ), compatible with the presence of silicates.

\section{Future perspectives}

Our analysis shows that the identification of cometary trails in in situ dust data may be possible even with a relatively small dust instrument. This suggests that it may be possible to identify impacts of cometary trail particles in the data sets of other space missions as well. The Ulysses spacecraft provided the longest continuous data set of in situ dust measurements in interplanetary space presently available: dust measurements were collected over 17 years while the spacecraft made three revolutions around the Sun (Grün et al. 1992; Krüger et al. 2010). We may be able to identify impacts of cometary trail particles in this data set as well because the spacecraft traversed the same regions of space up to three times. Given that the sensitive area of the Ulysses dust detector was about a factor of eight larger than the combined area of the Helios detectors, the search for cometary meteoroid trails in the Ulysses data set is promising and ongoing. Finally, the dust detector on board the New Horizons spacecraft 
(Horányi et al. 2008; Poppe et al. 2010) may reveal cometary trail crossings in the outer Solar System.

Large variations in the predicted dust fluxes from comet to comet have to be expected in the IMEX model because the ejection velocity, mass distribution, and dust production rate - all parameters of the model potentially as a function of time - likely vary for each comet and are not yet well constrained for many comets. This may be improved in the future for the comets found in our analysis, which may yield more reliable flux predictions.

The present IMEX model has a lower particle mass limit of $10^{-8} \mathrm{~kg}$. Future model extensions may include smaller particle sizes as well in order to cover particles that are more susceptible to solar radiation pressure. The trajectories of such smaller particles are expected to be offset from those of their bigger counterparts. Future simulations with such an extended model may give better agreement for the comets identified in our present analysis, and they may reveal additional comets to explain the Helios trail particle detections.

Many meteor streams and fireballs observed in the Earth's atmosphere were successfully traced back to their parent comets and asteroids (e.g. Jenniskens 2006). Extraterrestrial dust was collected in the Earth atmosphere by high-flying aircraft and its analysis in the laboratory provided a wealth of information (Jessberger et al. 2001, and references therein); however, their individual source bodies usually remain unknown. Only in very rare cases could "targeted" collections catch particles from a dedicated comet when the Earth crossed its trail, such as for example comet 26P/Grigg-Skjellerup (Busemann et al. 2008; Davidson et al. 2012). There have also been attempts to measure the particle composition of the induced meteors in the Earth atmosphere by ground-based observations (e.g. Toscano et al. 2013). However, these are strongly limited by contamination from atmospheric constituents. The in situ detection and analysis of cometary trail particles in space creates a new opportunity to remotely measure the composition of celestial bodies without the necessity to fly a spacecraft to the source objects.

The DESTINY ${ }^{+}$(Demonstration and Experiment of Space Technology for INterplanetary voYage with Phaethon fLyby and dUst Science) mission will be launched to the active nearEarth asteroid (3200) Phaethon in 2024 (Kawakatsu \& Itawa 2013; Arai et al. 2018). The DESTINY ${ }^{+}$Dust Analyzer (DDA; Kobayashi et al. 2018b) on board is an upgrade of CDA which very successfully investigated dust throughout the Saturnian system (Srama et al. 2011). The instrument will measure the composition of interplanetary and interstellar dust during the spacecraft's interplanetary journey to Phaethon as well as dust released from Phaethon during a close flyby at the asteroid. Recently, Phaethon's dust trail was identified in optical images obtained with the STEREO spacecraft (Battams et al. 2020).

We also performed IMEX simulations for the DESTINY ${ }^{+}$ mission based on the spacecraft trajectory presently available. Our results show that DESTINY ${ }^{+}$will traverse the trail of comet 45P/H-M-P three times in 2026 and 2027. If this is confirmed in the future for the real DESTINY ${ }^{+}$trajectory to be flown in space, this coincidence provides the interesting opportunity to compare the Helios spectra of likely trail particles with the high-resolution DDA spectra from the same source comet. Such a comparative study may also give new insights into the interpretation of the full set of 235 Helios mass spectra.

Other present or future space missions equipped with dust detectors include BepiColombo which has the Mercury Dust Monitor on board (MDM, Kobayashi et al. 2020). Even though MDM is partially obstructed by the heat shield of BepiColombo during the spacecraft's interplanetary passage to Mercury, the sensor may be able to detect particles in the trail of comet 2P/Encke en route to planet Mercury. The Martian Moons Exploration (MMX) mission will be launched to Phobos and Deimos in 2024, and the large-area $\left(\sim 1 \mathrm{~m}^{2}\right)$ dust impact detector on board may detect cometary trails en route to Mars (Kobayashi et al. 2018a; Krüger et al. 2019a). Furthermore, we encourage the Europa mission team with its SUrface Dust Analyzer (SUDA, Kempf 2018) to search for interplanetary dust and possible trails along its way to Jupiter. Finally, the JUpiter Icy moons Explorer (JUICE) mission (Witasse 2019) will not carry a dust sensor, but the radio plasma instrument might allow the detection of interplanetary dust particles. Plasma-wave instrument activation during dust trail crossing zones is recommended.

\section{Conclusions}

We re-analysed a subset of seven dust impacts measured in the 1970 s by the Helios dust instruments in the inner Solar System. The particles were originally identified by Altobelli et al. (2006) as potential cometary trail particles because of their clustering in a small region of space at a true anomaly angle of $135 \pm 1^{\circ}$ during several revolutions of Helios around the Sun. We modelled Helios traverses of cometary meteoroid trails with the IMEX dust streams in space model (Soja et al. 2015b), which simulates recently created cometary meteoroid streams in the inner Solar System.

The identification of potential cometary trail particles in the Helios data greatly benefitted from the clustering of trail traverses in a rather narrow region of space. We identified comets 45P/Honda-Mrkos-Pajdušáková and 72P/Denning-Fujikawa as the likely sources for a subset of four of the candidate trail particles. Using the Helios measurements in combination with the simulation results, we found spatial densities of approximately $10 \mu \mathrm{m}$ dust particles in the trails of these comets of $10^{-8}-10^{-7} \mathrm{~m}^{-3}$. Our analysis shows that trail particles are likely detectable with an in situ dust impact detector when the spacecraft traverses such a dense cometary dust trail. This creates the possibility to analyse celestial bodies remotely, without the necessity to fly a spacecraft close to or even land on the source objects.

Acknowledgements. The IMEX Dust Streams in Space model was developed under ESA funding (contract 4000106316/12/NL/AF - IMEX). We are grateful to the MPI für Sonnensystemforschung, Chiba Institute of Technology and the University of Stuttgart for their support. H. Kimura gratefully acknowledges support by the Grants-in-Aid for Scientific Research (KAKENHI number 19H05085) of the Japan Society for the Promotion of Science (JSPS). We thank an anonymous referee whose comments improved the presentation of our results.

\section{References}

Agarwal, J., Müller, M., \& Grün, E. 2007, Space Sci. Rev., 128, 79

Agarwal, J., Müller, M., Reach, W. T., et al. 2010, Icarus, 207, 992

A'Hearn, M. F., Millis, R. C., Schleicher, D. O., Osip, D. J., \& Birch, P. V. 1995, Icarus, 118, 223

Altobelli, N., Grün, E., \& Landgraf, M. 2006, A\&A, 448, 243

Altobelli, N., Moissl, R., Krüger, H., Landgraf, M., \& Grün, E. 2004, Planet. Space Sci., 52, 1287

Arai, T., Kobayashi, M., Ishibashi, K., et al. 2018, in Lunar and Planetary Institute Science Conference Abstracts, Vol. 49, Lunar and Planetary Institute Science Conference Abstracts, 2570

Arendt, R. G. 2014, AJ, 148, 135

Auer, S. 2001, in Interplanetary Dust, eds. E. Grün, B. A. S. Gustafson, S. F. Dermott, \& H. Fechtig (Berlin, Heidelberg, New York: Springer Verlag), 385 Battams, K., Knight, M. M., Kelley, M. S. P., et al. 2020, ApJS, 246, 64

Beech, M. 2001, MNRAS, 327, 1201

Beech, M., Nikolova, S., \& Jones, J. 1999, MNRAS, 310, 168

Burns, J. A., Lamy, P. L., \& Soter, S. 1979, Icarus, 40, 1 
Busemann, H., Nguyen, A. N., Nittler, L. R., et al. 2008, Geochim. Cosmochim. Acta Suppl., 72, A124

Christou, A., Vaubaillon, J., Withers, P., Hueso, R., \& Killen, R. 2019, ExtraTerrestrial Meteors, eds. G. O. Ryabova, D. J. Asher, \& M. J. Campbell-Brown (Cambridge: Cambridge University Press), 119

Crifo, J. F., \& Rodionov, A. V. 1997, Icarus, 127, 319

Davidson, J., Busemann, H., \& Franchi, I. A. 2012, Meteoritics Planet. Sci., 47, 1748

Dietzel, H., Eichhorn, G., Fechtig, H., et al. 1973, J. Phys. E Sci. Instrum., 6, 209

Divine, N., \& Newburn, R. L., J. 1987, A\&A, 187, 867

Eichhorn, G. 1978a, Planet. Space Sci., 26, 463

Eichhorn, G. 1978b, Planet. Space Sci., 26, 469

Fechtig, H., Grün, E., \& Kissel, J. 1978, in Cosmic Dust, ed. J. A. M. McDonnell (Wiley), 607

Grün, E. 1981, Physical and chemical characteristics of interplanetary dust particles. Measurements by the micrometeoroid experiment on board HELIOS, Tech. rep., Final report, June 1981, Max-Planck-Inst. für Kernphysik, Heidelberg, (Germany, F.R.)

Grün, E., Pailer, N., Fechtig, H., \& Kissel, J. 1980, Planet. Space Sci., 28, 333

Grün, E., Fechtig, H., Kissel, J., et al. 1992, A\&AS, 92, 411

Grün, E., Gustafson, B. E., Mann, I., et al. 1994, A\&A, 286, 915

Grün, E., Baguhl, M., Hamilton, D. P., et al. 1995, Planet. Space Sci., 43, 941

Grün, E., Krüger, H., Graps, A., et al. 1998, J. Geophys. Res., 103, 20011

Güttler, C., Mannel, T., Rotundi, A., et al. 2019, A\&A, 630, A24

Hasegawa, I. 2001, in Meteoroids 2001 Conference, ed. B. Warmbein, ESA SP, 495, 55

Hillier, J. K., Green, S. F., McBride, N., et al. 2007, Icarus, 190, 643

Hirai, T., Cole, M. J., Fujii, M., et al. 2014, Planet. Space Sci., 100, 87

Hirn, A., Albin, T., Apathy, I., et al. 2016, A\&A, 591, A93

Horányi, M., Hoxie, V., James, D., et al. 2008, Space Sci. Rev., 140, 387

Ishiguro, M., Sarugaku, Y., Ueno, M., et al. 2007, Icarus, 189, 169

Ishiguro, M., Kuroda, D., Hanayama, H., et al. 2016, AJ, 152, 169

Jenniskens, P. 2006, Meteor Showers and their Parent Comets (Cambridge University Press)

Jessberger, E. K., Stephan, T., Rost, D., et al. 2001, in Interplanetary Dust, eds. E. Grün, B. A. S. Gustafson, S. F. Dermott, \& H. Fechtig (Berlin, Heidelberg, New York: Springer Verlag), 253

Jones, M. H., Bewsher, D., \& Brown, D. S. 2013, Science, 342, 960

Jones, M. H., Bewsher, D., \& Brown, D. S. 2017, Icarus, 288, 172

Jorda, L., Crovisier, J., \& Green, D. W. E. 2008, in Asteroids Comets Meteors, 1405,8046

Kawakatsu, Y., \& Itawa, T. 2013, Adv. Astronaut. Sci., 146, 12

Kempf, S. 2018, in European Planetary Science Congress, EPSC2018-462
Kimura, H., \& Mann, I. 1999, in Meteroids 1998, eds. W. J. Baggaley, \& V. Porubcan (Astronomical Institute of the Slovak Academy of Sciences), 283 Kimura, H., Hilchenbach, M., Merouane, S., Paquette, J., \& Stenzel, O. 2020a, Planet. Space Sci., 181, 104825

Kimura, H., Wada, K., Yoshida, F., et al. 2020b MNRAS, 496, 1667

Kobayashi, M., Krüger, H., Senshu, H., et al. 2018a, Planet. Space Sci., 156, 41

Kobayashi, M., Srama, R., Krüger, H., Arai, T., \& Kimura, H. 2018b, in Lunar and Planetary Institute Science Conference Abstracts, 49, 2050

Kobayashi, M., Shibata, H., Nogami, K., et al. 2020, Space Sci. Rev., submitted

Koschny, D., Soja, R. H., Engrand, C., et al. 2019, Space Sci. Rev., 215, 34

Krüger, H., Dikarev, V., Anweiler, B., et al. 2010, Planet. Space Sci., 58, 951

Krüger, H., Seidensticker, K. J., Fischer, H. H., et al. 2015, A\&A, 583, A15

Krüger, H., Kobayashi, M., Sasaki, S., et al. 2019a, in EPSC-DPS Joint Meeting 2019, Vol. 2019, EPSC-DPS2019-112

Krüger, H., Strub, P., Altobelli, N., et al. 2019b, A\&A, 626, A37

Leinert, C., \& Moster, B. 2007, A\&A, 472, 335

Levasseur-Regourd, A.-C., Agarwal, J., Cottin, H., et al. 2018, Space Sci. Rev., 214, 64

Pokorný, P., \& Kuchner, M. 2019, Astrophys. Lett., 873, L16

Poppe, A., James, D., Jacobsmeyer, B., \& Horányi, M. 2010, Geophys. Res. Lett., 37, L11101

Reach, W. T., Kelley, M. S., \& Sykes, M. V. 2007, Icarus, 191, 298

Sato, H., \& Williams, G. V. 2014, Minor Planet Electronic Circulars, 2014-M11

Soja, R. H., Herzog, J. T., Sommer, M., et al. 2015a, in International Meteor Conference Mistelbach, Austria, eds. J.-L. Rault, \& P. Roggemans, 66

Soja, R. H., Sommer, M., Herzog, J., et al. 2015b, A\&A, 583, A18

Soja, R. H., Grün, E., Strub, P., et al. 2019, A\&A, 628, A109

Sommer, M., Yano, H., \& Srama, R. 2020, A\&A, 635, A10

Springmann, A., Howell, E. S., Harmon, J. K., et al. 2017, in AAS/Division for Planetary Sciences Meeting Abstracts \#49, AAS/Division for Planetary Sciences Meeting Abstracts, 305.06

Srama, R., Kempf, S., Moragas-Klostermeyer, G., et al. 2011, CEAS Space J., 2 , 3

Sykes, M. V., Lebofsky, L. A., Hunten, D. M., \& Low, F. 1986, Science, 232, 1115

Srama, R., Ahrens, T. J. Altobelli, N., Auer, S., et al. 2004, Space Sci. Rev., 114, 465

Sykes, M. V., \& Walker, R. G. 1992, Icarus, 95, 180

Toscano, F. M., Madiedo, J. M., Trigo-Rodríguez, J. M., et al. 2013, in Lunar and Planetary Science Conference, 1066

Vaubaillon, J., \& Christou, A. A. 2006, A\&A, 451, L5

Witasse, O. 2019, in EPSC-DPS Joint Meeting 2019, Vol. 2019, EPSC-DPS2019400

Yano, H., Hirai, T., Okamoto, C., Tanaka, M., \& Fujii, M. 2014, in 40th COSPAR Scientific Assembly, Vol. 40, B0.5-6-14 